\title{
A Comparative Analysis of WiMax System with Partial Transmit Sequence (PTS) and Low Complexity PTS
}

\author{
Kavita Dave', Anand Jain ${ }^{2}$ \\ ${ }^{1}$ PG Student, ECE Department, Aravali Institute of Technical Studies, Udaipur \\ ${ }^{2}$ HOD, ECE Department, Aravali Institute of Technical Studies, Udaipur
}

\begin{abstract}
Partial transmit sequence (PTS) is one of the most well-known peak-to-average power ratio (PAPR) reduction techniques proposed for orthogonal frequency-division multiplexing (OFDM) systems. The main drawbacks of the conventional PTS (C-PTS) are high computational complexity and transmission of several side information bits. A new PTS with simple detector is proposed in this paper to deal with these drawbacks of C-PTS. The candidates can be generated through cyclically shifting each subblock sequence in time domain and combining them in a recursive manner. At the receiver, by utilizing the natural diversity of phase constellation for different candidates, the detector can successfully recover the original signal without side information. Numerical simulation shows that the proposed scheme performs very well in terms of PAPR. The probability of detection failure of the side information demonstrates that the detector could work without any side information with high reliability. The proposed scheme achieves almost the same bit error rate (BER) performance as the C-PTS with perfect side information, under both additive white Gaussian noise (AWGN) channel and Rayleigh fading channel.
\end{abstract}

Keywords: OFDM, PAPR, SQNR, clipping, PTS, DFT.

\section{Introduction}

Orthogonal frequency division multiplexing (OFDM) is an attractive technique for wireless high-rate data transmission due to the minimizing effect over frequency-selective fading channels [1]. As such, OFDM has been chosen for European Digital Audio Broadcasting (DAB), Digital Video Broadcasting (DVB), WLAN standards (802.11), WiMax (802.16) and is being considered for the long term evolution of 3GPP. However, OFDM has some drawbacks in the transmission system. One of the major problems of the OFDM system is that OFDM signal has higher peak toaverage-power ratio (PAPR) than single carrier signal because OFDM signal is the sum of many narrowband signals in the time domain [2]. The high PAPR can cause inter-modulation and out-of-band radiation due to power amplifier nonlinearity. In order to combat the problem, the transmission amplifier must operate within its linear region to prevent spectral distortion and the degradation of the bit error rate (BER). High linearity normally implies low efficiency and large power dissipation, which is prohibitive for use in portable wireless applications [3]. Therefore, it is highly desirable to reduce the PAPR of an OFDM signal. OFDM PAPR reduction has been a subject of intense research in the past decade. Many methods have been proposed including clipping of the OFDM signal [4], coding techniques [5], active constellation extension (ACE) [6], companding transform [7], [8], tone reservation (TR) [9], [10], tone injection (TI) [11], partial transmit sequence (PTS) [12]-[16], selective mapping (SLM) [16]-[20] and various combinations of the above. Among them, SLM and PTS are two promising techniques because they are simple to implement, no distortion in the transmitted signal and can significantly improve the statistics of the PAPR. However, the conventional SLM and PTS suffer from higher computational complexity due to several -dimensioned inverse fast Fourier transform (IFFT) operations, where is the number of subcarriers. At the same time in order to recover the original OFDM signal successfully, the transmitter has to send the selected signal index, called side information, to the receiver using extra subcarrier.

It will degrade the OFDM system's spectrum efficiency. Worst still, the BER performance of the OFDM systems can possibly be degraded significantly since any error in the detection of side information can damage the entire data block. Several proposals that suggested techniques for eliminating the need of side information have appeared in the literature [21]-[25]. However, these proposed techniques rely on some reference symbols that increase the transmission power or with the potential of higher complexity for the detector. We are motivated to deal with the two drawbacks of the conventional PTS togethercomplexity and side information. In this paper, interleaved PTS making use of the combination of cyclically shifting sub-block sequences is proposed to generate new candidates. Our scheme can reduce the computational complexity without any side information. The advantages of the proposed scheme are described as follows. Firstly, with the interleaved partition method, reduction of computational complexity can be achieved by using Cooley-Tukey FFT algorithm [26]. Secondly by utilizing cyclically shifted subblock sequences and IFFT property, the proposed scheme will generate a set of candidates with different phase constellation without multiplication according to the different shifting number of the subblock. furthermore, we can utilize the reduced complexity recursive method in our previous work to further decrease the computational complexity [14]. The detector at the receiver can distinguish which candidate has been transmitted through the minimum Hamming distances of the receiver signals (after FFT) to the phase constellation for each sub-block without side 


\section{International Journal of Science and Research (IJSR) \\ ISSN (Online): 2319-7064 \\ Index Copernicus Value (2013): 6.14 | Impact Factor (2015): 6.391}

information. Because the cyclically shifting operates on each subblock individually, hence the overall detected number only increases linearly with the number of sub-block rather than exponentially as in C-PTS.

\section{PTS in OFDM for PAPR Reduction}

Two more distortion-less PAPR reduction techniques namely partial transmit sequence (PTS) [11] and selective mapping (SLM) [12] are also proposed in the literature. In PTS scheme all the subcarriers are partitioned into multiple disjoint sub blocks and then each of the sub blocks is multiplied by a set of rotating phase factors and combined to achieve a signal with lowest PAPR. In SLM, parallel data signal of length $\mathrm{N}$ is multiplied by a predetermined set of $\mathrm{U}$ phase vectors of length $\mathrm{N}$ andgenerates $\mathrm{U}$ alternative signals. Out of $U$ alternative signals, one of them with the least PAPR is selected for transmission. In both of the schemes the information about the phase factors by which these sub blocks/data symbols are multiplied, needs to be conveyed to the receiver and it is known as side information (SI).

The SI has the highest importance because it is used to recover the original data signal. If SI gets corrupted then entire OFDM symbol block can be damaged and error performance of SLM- and PTS-OFDM system degrades severely. In PTS technique, if the number of sub blocks increases then it not only increases computational complexity for selecting the optimum set( provide least possible PAPR) of phase sequence but also increases the amount of SI to be conveyed to the receiver. The SI results loss of data rate in OFDM system. Similarly in SLM-OFDM systems as the number of alternative OFDM signal increases, the number of bits required to encode the side information also gets increased, which results in data rate loss.

The SI bits are extremely important for data recovery and it may be necessary to allocate few redundant bits to ensure accurate recovery of SI, but this operation will further increases the loss of data rate in OFDM system. Many schemes for embedding the SI have been proposed in [13][14] for PTS-OFDM systems. In [15]-[17] many SI embedding schemes have been proposed for SLM-OFDM system. These schemes [13]-[17] embed SI in the OFDM signal without using any extra bit. At the receiver, SI is extracted from the received OFDM signal, and decoded to obtain the information about the phase factor used at the transmitter to minimize PAPR. The demodulated signal is multiplied by the reciprocal of recovered phase factors, due to which the computational complexity at the receiving end gets increased. In many of the SI embedding schemes, the SI detection at lower values of SNR is very poor, due to which error performance of the OFDM system degrades severely.

Existing SI embedding schemes [13]-[17] eliminates the requirement of SI transmission but these suffer from one drawback or the other, whether in terms of computational complexity, poor PAPR reduction capability or incorrect SI detection. In [18], Zhou et al. proposed MPSM-PTS scheme which extends the QPSK constellation points to disjoint points of 16-QAM constellation and eliminates the requirement of side information. The MPSM-PTS scheme [18] is completely free from SI, i.e. extraction of SI from the received signal is not required. Hence the receiver structure of the scheme proposed in [18] is computationally less complex.

In wireless standards like LTE, OFDM is used in downlink, where mobile station acts as receiver. The mobile stations have limited computational resources; therefore, a PAPR reduction scheme with less computational complexity at receiving end will be more beneficial. As discussed above, the schemes proposed in [13]-[17] have computationally complex receiver in comparison to the schemes proposed in [13], [14]. Hence, MPSM-PTS scheme is a viable choice for PTS-OFDM system.

\section{Proposed PTS Scheme and Detector}

\section{A. Proposed PTS Scheme}

The aim of the proposed PTS scheme is to lower the complexity of the transmitter and detector while the receiver can recover the original signal without side information. It is shown in ref [12] that, though with the worst PAPR performance when compared with the pseudo-random and adjacent partition method, the interleaved partition method can reduce the computational complexity by using CooleyTukey FFT algorithm. Ref [15] proves that the candidates in the conventional interleaved partitioning PTS scheme are not fully independent, which leads to performance inferior to the adjacent partitioning PTS scheme. After enhancing the independence among the candidates, the PAPR performance of the amended interleaved partitioning PTS scheme can be improved to achieve similar performance as the adjacent partitioning PTS scheme. The result in [15] inspirits us using the interleaved partition method in PTS scheme rather than pseudo-random and adjacent partition method considering the tradeoff of complexity and PAPR performance.

Below is the implemented graphical user interface in Matlab as shown below:- 
International Journal of Science and Research (IJSR)

ISSN (Online): 2319-7064

Index Copernicus Value (2013): 6.14 | Impact Factor (2015): 6.391

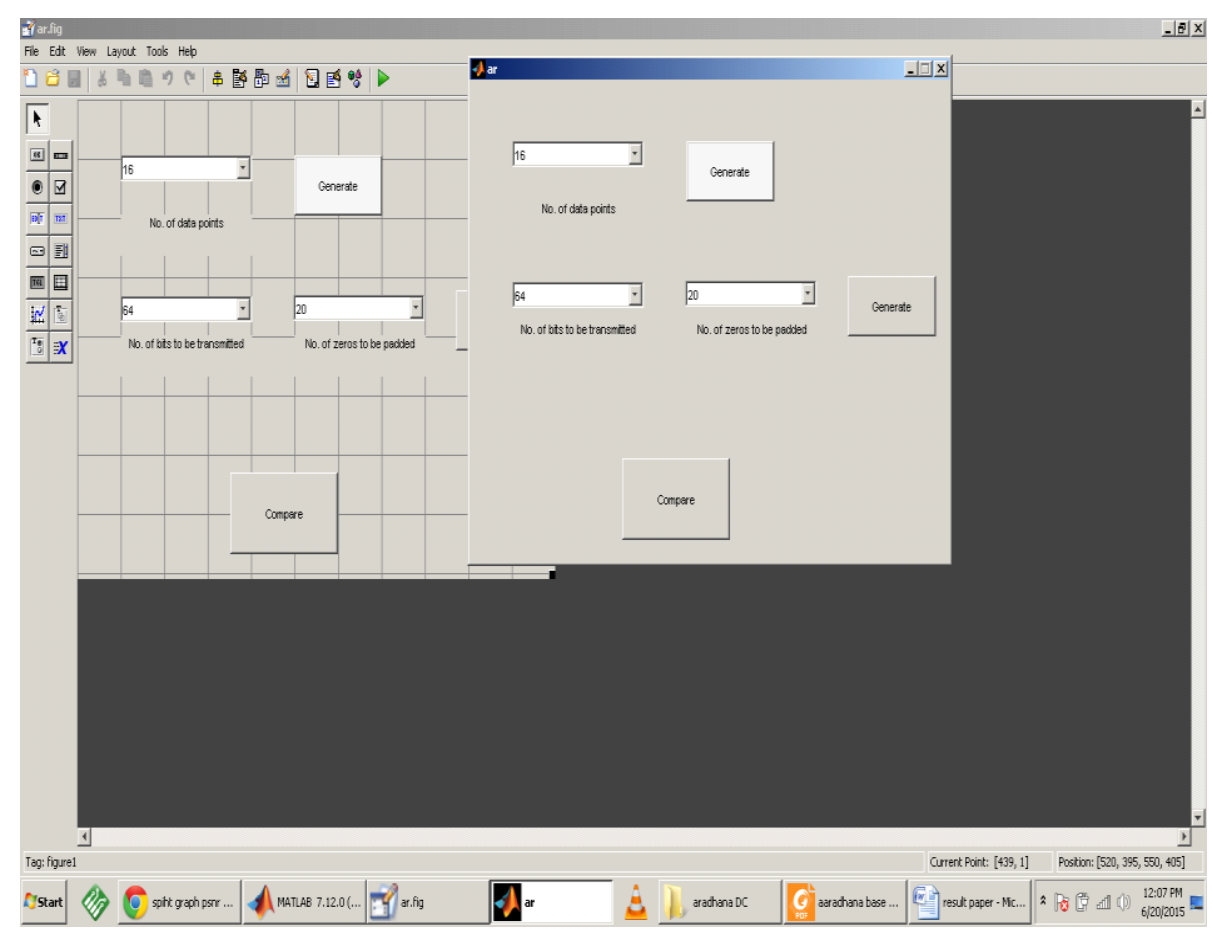

Figure 1: Complete GUI built using Matlab

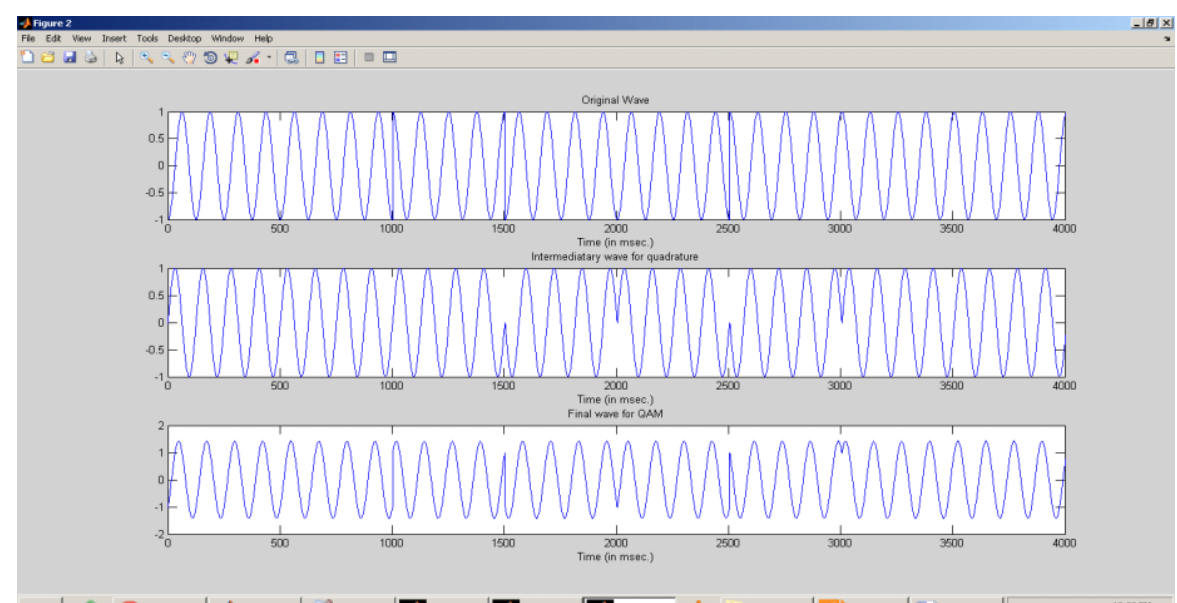

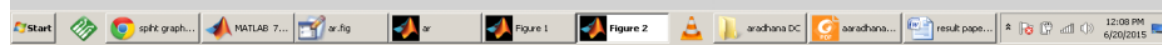

Figure 2: Waveform showing various modulations performed

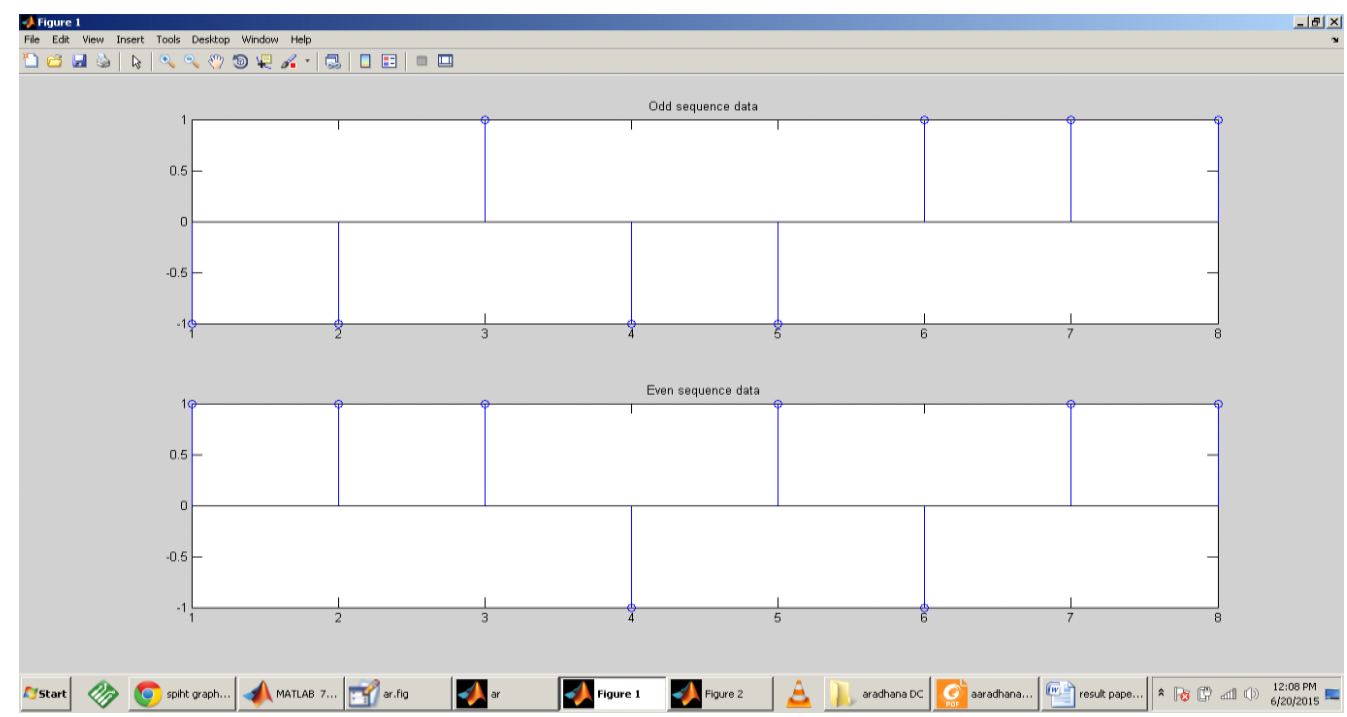

Figure 3: Graph showing ODD and EVEN sequences for OFDM signal

\section{Volume 5 Issue 6, June 2016} www.ijsr.net

Licensed Under Creative Commons Attribution CC BY 
International Journal of Science and Research (IJSR)

ISSN (Online): 2319-7064

Index Copernicus Value (2013): 6.14 | Impact Factor (2015): 6.391

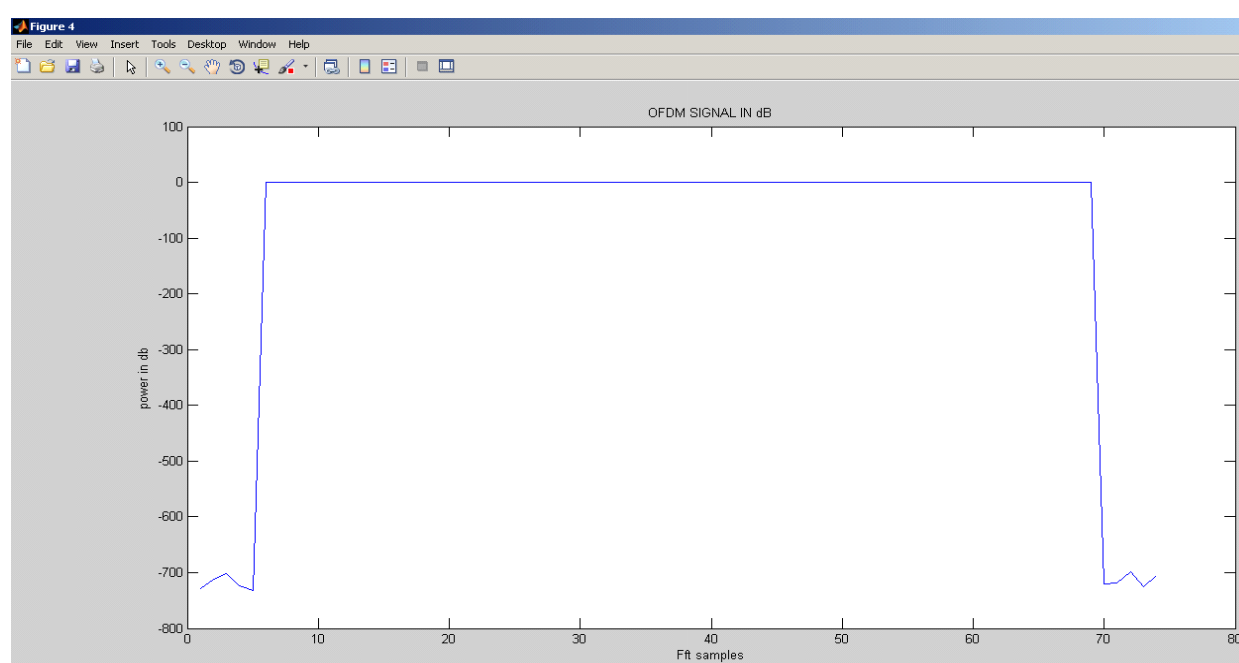

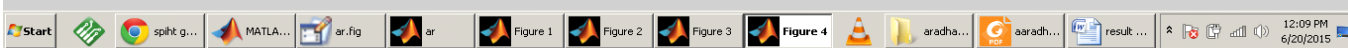

Figure 4: Graph showing power spectrum in $\mathrm{dB}$

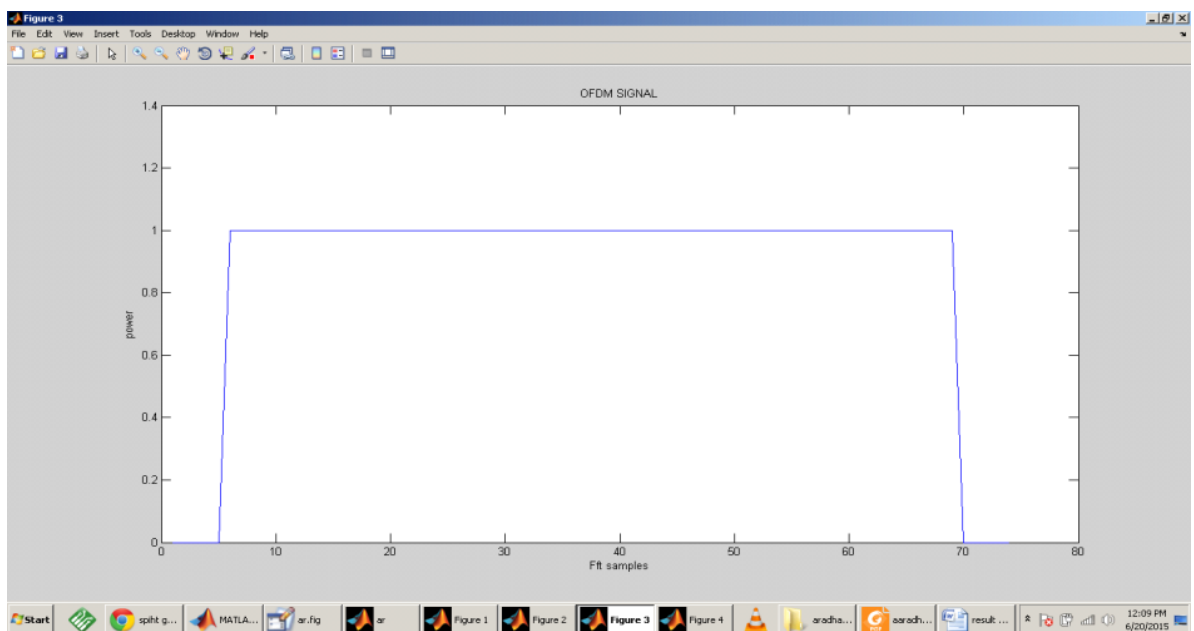

Figure 5: Graph showing linear power spectrum

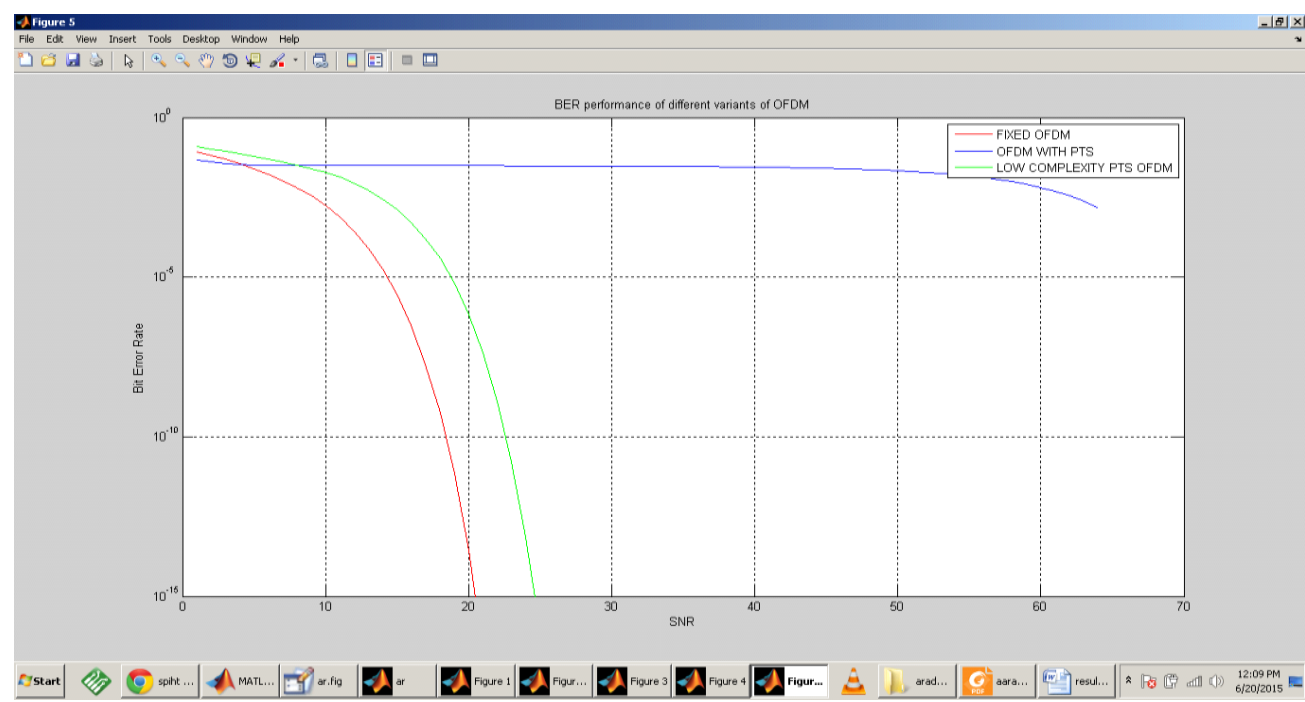

Figure 6: Graph showing comparative analysis for $\mathrm{N}=64$

Volume 5 Issue 6, June 2016 www.ijsr.net

Licensed Under Creative Commons Attribution CC BY 


\section{International Journal of Science and Research (IJSR) \\ ISSN (Online): 2319-7064}

Index Copernicus Value (2013): 6.14 | Impact Factor (2015): 6.391

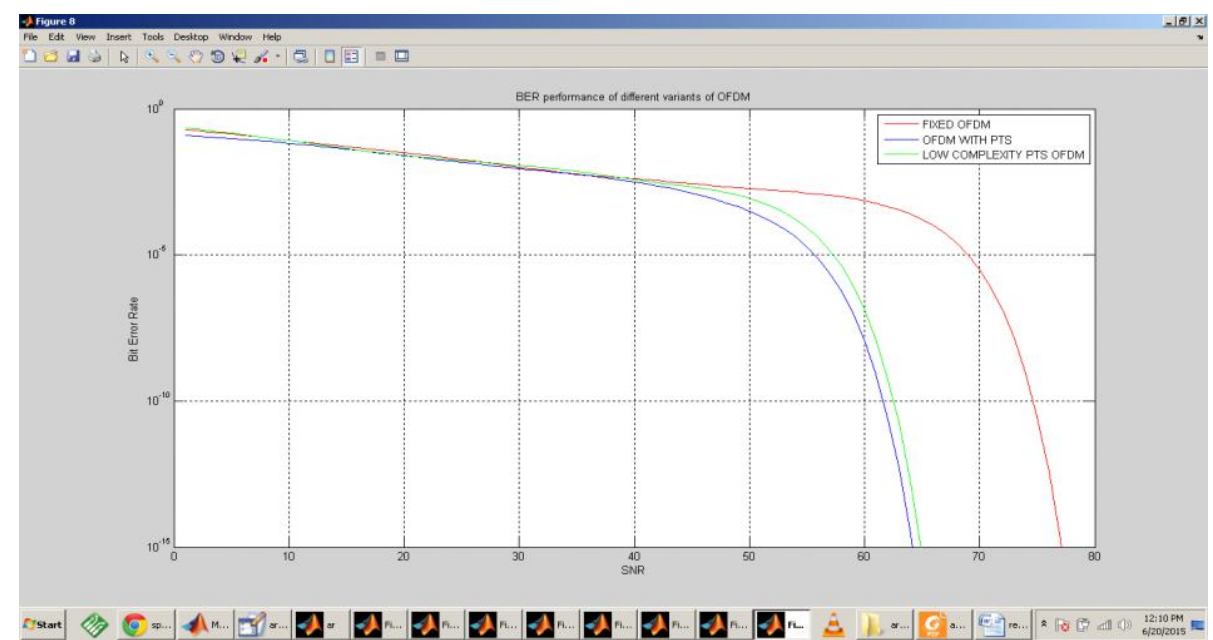

Figure 7: Graph showing comparative analysis for $\mathrm{N}=1024$

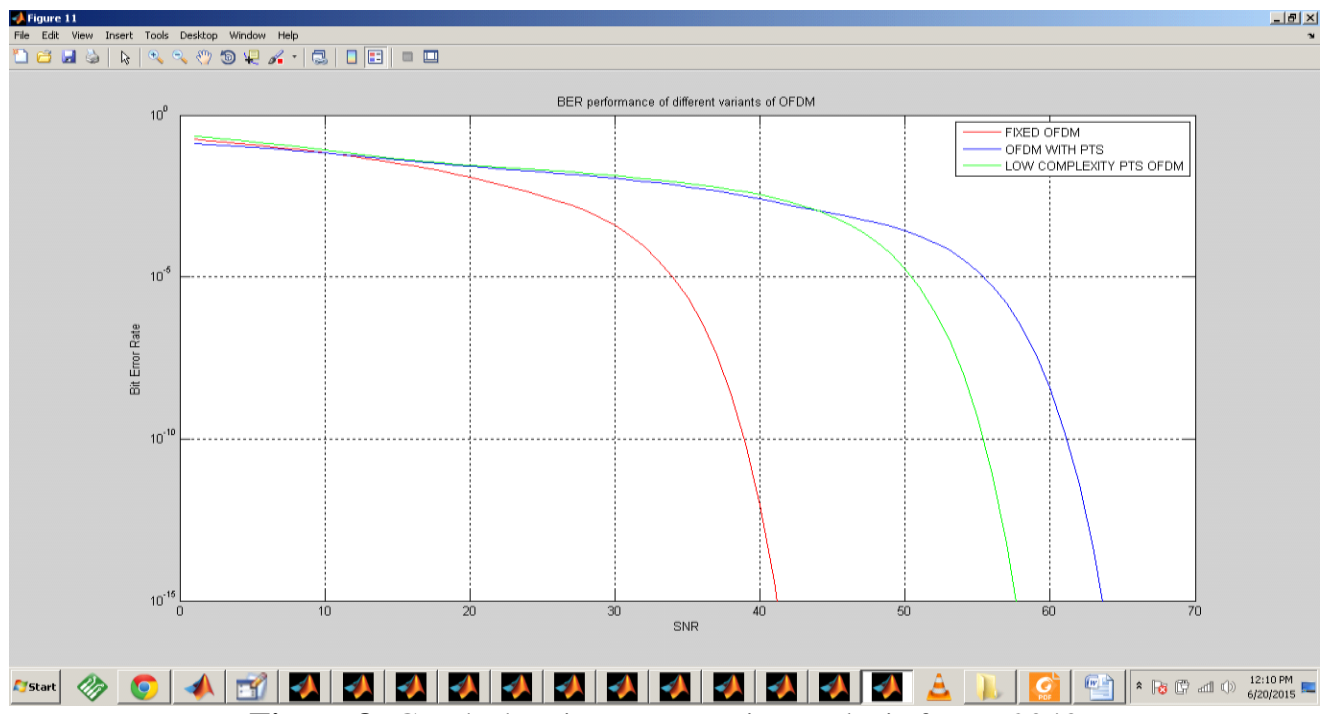

Figure 8: Graph showing comparative analysis for $\mathrm{N}=2048$

\section{Conclusion}

An interleaved partitioning PTS scheme making use of the recursive combination of cyclically shifting sub-block sequences and linear property of IFFT is presented in this paper. The proposed scheme will lower the complexity without side information. From the aspect of complexity, firstly, the Cooley-Tukey FFT can be implemented because of the interleaved partition method. Secondly, no multiplication is performed by the cyclically shifting. Thirdly, the reduced complexity recursive method is used to generate new candidates. Finally, the overall detected number at the detector increases linearly with the number of sub-blocks rather than exponentially with the increase of candidates. By utilizing cyclically shifting of the sub-block sequence, a set of candidates with different phase constellation will be generated according to the different shifting number of the subblock. As such, the detector can distinguish which candidates had been transmitted without any side information. Cyclically shifted sub-block sequences also increase the independence and the total number of candidates compared with the conventional PTS scheme. Simulation results have shown that the proposed scheme is reliable to estimate the selected shifting number of each subblock. As a result, it achieves almost the same BER performance as the conventional PTS scheme but without any side information both in AWGN and rayleigh fading channels. It may be worthwhile to further study the performance of the proposed PTS in frequency selective channel without perfect channel information in the future.

\section{References}

[1] Wu Y., Zou W. Y., "Orthogonal frequency division multiplexing: A multi-carrier modulation scheme", IEEE Trans. Consumer Electronics, vol. 41, no. 3, pp. 392-399, Aug. 1995.

[2] Van Nee R., Prasad R., "OFDM for Wireless Multimedia Communications", Artech House, 2000.

[3] Jiang T., Wu Y., "An Overview: Peak-to-average power ratio reduction techniques for OFDM signals", IEEE Trans. Broadcasting, vol. 54, No. 2, pp. 257-268, Jun. 2008.

[4] Armstrong, J., "Peak-to-average power reduction for OFDM by repeated clipping and frequency domain filtering," Electronics Letters, vol. 38, No. 5, pp. 246247, Feb. 2002.

[5] Wang X. B., Tjhung T. T., Ng C. S., "Reduction of peak-to-average power ratio of OFDM system using a companding technique", IEEE Transaction on Broadcasting, vol. 45, No. 3, pp. 303-307, Sept. 1999.

\section{Volume 5 Issue 6, June 2016} www.ijsr.net 


\section{International Journal of Science and Research (IJSR) \\ ISSN (Online): 2319-7064 \\ Index Copernicus Value (2013): 6.14 | Impact Factor (2015): 6.391}

[6] Jiang T., Yang Y., Song Y., "Exponential companding transform for PAPR reduction in OFDM systems", IEEE Transaction on Broadcasting, vol. 51, No. 2, pp. 244-248, June 2005.

[7] Huang J. L., Zheng J., Letaief K. B., Gu J., "Companding Transform for Reduction in Peak-toAverage Power Ratio of OFDM Signals", IEEE Transaction on Wireless Communications, Vol. 03, No. 6, pp. 2030-2039, November 2004.

[8] Sulaiman A. A., Ehab F. B., Mohamed Darwish A. E., "Linear Companding Transform for the Reduction of Peak-to-Average Power Ratio of OFDM Signals", IEEE Transaction Broadcasting, Vol. 55, No. 1, pp. 195-200, March 2009.

[9] Hou, J., Ge J. H., Li J., "Trapezoidal companding scheme for peak-to-average power ratio reduction of OFDM signals," Electronics Letters, Vol. No. pp 1349 1351, Dec. 2009.

[10] Jeng S. S., Chen J. M., "Efficient PAPR reduction in OFDM system based on a companding techniques with trapezium distribution", IEEE Transaction on Broadcasting, Vol. 57, No. 2, pp. 291-298, June 2011.

[11] Muller, S. H., Huber, J. B., "OFDM with reduced peak-to-average power ratio by optimum combination of partial transmit sequences", Electronics Letters, Vol. 33, No. 5, pp. 36-69, Feb. 1997.

[12] Bäuml R. W., Fischer R. F. H., Huber J. B., "Reducing the peak-to average power ratio of multicarrier modulation by selected mapping", Electronics Letters., Vol. 32, No. 22, pp. 2056-2057, Oct. 1996.

[13] Jayalath A. D. S., Tellambura C., "SLM and PTS peakpower reduction of OFDsignals without side information," IEEE Transaction on Wireless Communication., Vol. 4, No. 5, pp. 2006-2013, Sep. 2005.

[14] Cimini Jr. L. J., and Sollenberger N.R., "Peak-toAverage Power Ratio Reduction of an OFDM Signal Using Partial Transmit Sequences with embedded side information”, Proc IEEE GlobeComm'00, ,pp 740-750, Nov. 2000.

[15]Breiling M., Muller-Weinfurtner S., Huber J., "SLM peak power reduction without explicit side information", IEEE Communication Letters, Vol. 5, No. 6, pp. 239-241, Jun. 2001.

[16] Le Goff S. Y.,.Al-Samahi S.S, Boon K. K., Tsimenidis C. C., Sharif B. S., "Selected Mapping without Side Information for PAPR reduction in OFDM", IEEE Transaction on Wireless Communications, Vol. 8, No. 7, pp. 3320-3325, July 2009.

[17] Irukulapati, N.V., Chakka V. K., Jain A., "SLM based PAPR reduction of OFDM signal using new phase sequence", Electronics Letters, Vol. 45, No. 24, pp. 1231-1232, Nov. 2009

[18]Zhou, Y. , Jiang, T., “A Novel Multi-Point Square Mapping Combined with PTS to Reduce PAPR of OFDM Signals without Side Information", IEEE Transaction on Broadcasting, Vol. 55, No. 4, pp. 831835, Dec. 2009.

[19]Zhao Y., Häggman S. G., "Intercarrier interference selfcancellation scheme for OFDM mobile communication systems," IEEE Transaction on Communications, Vol. 49, No. 7, pp. 1185-1191, July 2001.
[20] Sathananthan K., Rajatheva, R. M. A. P., “Analysis of OFDM in the presence of Frequency Offset and a method to reduce performance degradation", Proc. of Global telecommunications conference'00, San Francisco, USA, Vol. 1 pp.72-76, Nov. 2000.

[21] Seyedi A., Saulnier, G.J., "General ICI Selfcancellation scheme for OFDM systems", IEEE Transaction on Vehicular Technology, Vol. 54, No. 1 January 2005.

[22] Yeh H.G., Chang, Y.-K., Hassibi B., "A scheme for canceling intercarrier interference using conjugate transmission in multicarrier communication systems", IEEE Transaction on Wireless Communication, Vol. 6, No. 1, pp. 3-7, Jan. 2007.

[23] Wang C. L., Huang Y. C., "Intercarrier interference cancellation using general phase rotated conjugate transmission for OFDM systems", IEEE Transaction on Communications, Vol. 58, No. 3, March 2010. 